\title{
Síntese de nanopartículas de magnetita com diâmetro controlado visando aplicações em hipertermia
}

Paula Morais* (Graduada em Eng. Química na Universidade Federal Rural do Semi-Árido - UFERSA);

Dr. Sc Marco Morales (Prof. Ad. do DFTE na Universidade Federal do Rio Grande do Norte - UFRN);

Mayra Monteiro (Graduada em Eng. Química na Universidade Federal Rural do Semi-Árido - UFERSA);

Karyn Oliveira (Graduanda em Eng. Química na Universidade Federal Rural do Semi-Árido - UFERSA);

* Email: paula.morais.lima@gmail.com

\section{Resumo:}

Nesta apresentação mostraremos um método para a preparação de nanopartículas de magnetita com diâmetro e densidade de partículas controladas. As nanopartículas foram sintetizadas na matriz da quitosana, dois conjuntos de amostras foram preparados. A densidade das nanopartículas foi aumentada controlando a quantidade de reagentes. Medidas de difração de raios-X mostram que as nanopartículas têm parâmetro de rede de 0,839 Á e apresentam diâmetro médio de 12,6 nm. Imagens de microscopia eletrônica de transmissão mostraram partículas monodispersas com distribuição de tamanho Log-Normal muito estreita. O parâmetro de rede obtido mostra que as nanopartículas são de elevada pureza. Medidas de espectroscopia Mössbauer, feitas em temperatura ambiente, mostraram espectros devido a partículas bloqueadas magneticamente, sendo esse efeito devido principalmente à interação magnética entre as nanopartículas.

\section{Pallavras-chave:}

Hipertermia magnética; magnetismo; magnetita. 


\section{Introdução}

Segundo Arantes (2010), existe um grande interesse no estudo de nanopartículas magnéticas, devido à grande quantidade de aplicações tecnológicas em que elas podem ser usadas. Na área biomédica há uma grande quantidade de técnicas envolvendo o uso de nanopartículas magnéticas, tais como, imagens por ressonância magnética, tratamentos médicos como a hipertermia, vectorização magnética de fármacos neoplásicos, imobilização de enzimas, etc.

Ferrofluidos de nanopartículas de magnetita podem desenvolver coercividade diminuído as distâncias entre as partículas de modo que elas interajam magneticamente. Foi observado que a interação dipolar magnética entre as partículas pode deslocar a temperatura de bloqueio para valores maiores, e que a quantidade de calor liberado pelas partículas é proporcional à área contida em um ciclo de histerese. Assim, aumentando a densidade do ferrofluido será possível aumentar a coercividade e também a taxa de liberação de calor (Zhang, 2010).

A aplicação de diferentes óxidos de ferro para procedimentos como a hipertermia magnética está ganhando grande aceitação em diversos tipos de práticas, explorando as duas maiores vantagens desses óxidos, sua baixa toxicidade (biocompatibilidade) em seres humanos e seu alto valor de dipolo magnético. A hipertermia com partículas de magnetita é uma técnica utilizada no tratamento do câncer, que consiste no aquecimento dos tecidos malignos com aplicação de um campo magnético alternado sobre estas partículas. Esta técnica tem sido estudada em ferrofluidos de baixa densidade.

As nanopartículas são aquecidas através da relaxação de Néel ou de Brown, o calor gerado é transmitido para as células que se encontram na vizinhança. As células cancerosas geralmente perecem na faixa de $43-45^{\circ} \mathrm{C}$, pois sua irrigação via vasos sanguíneos é ineficiente, enquanto células normais não são danificadas na mesma faixa de temperatura. (Souza, 2011).

\section{Objetivos}

\subsection{Objetivo Geral}

Desenvolver um método para preparação de nanopartículas de magnetita com diâmetro controlado e densidade de partículas variável visando aplicações em hipertermia.

\subsection{Objetivos Específicos}

- Preparar nanopartículas de magnetita com diâmetro controlado;

- Desenvolver um método para aumentar a concentração das partículas visando facilitar as interações dipolares magnéticas entre elas;

- Caracterizar fisicamente as nanopartículas de magnetita.

\section{Metodologia}

As amostras foram preparadas em temperatura ambiente. Para a síntese das nanopartículas, quantidades iguais de $\mathrm{Fe} \mathrm{2+} \mathrm{e} \mathrm{Fe} \mathrm{3+} \mathrm{em} \mathrm{miligramas} \mathrm{(mg)} \mathrm{foram} \mathrm{misturadas} \mathrm{a} 10 \mathrm{~mL}$ da solução de quitosana. A quantidade de quitosana foi constante em todas as amostras. Usaram-se quantidades iguais dos ferros com o intuito de compensar o efeito de oxidação do Fe 2+, já que durante a preparação a amostra interage com o oxigênio e este ferro pode oxidar. As massas usadas para os reagentes utilizados estão mostrados na tabela a seguir:

\subsection{Método de síntese das amostras}

As amostras foram preparadas em temperatura ambiente. Para a síntese das nanopartículas, quantidades iguais de Fe 2+ e Fe 3+ em miligramas (mg) foram misturadas a $10 \mathrm{~mL}$ da solução de quitosana. A quantidade de quitosana foi constante em todas as amostras. Usaram-se quantidades iguais dos ferros com o intuito de compensar o efeito de oxidação do Fe 2+, já que durante a preparação a amostra interage com o oxigênio e este ferro pode oxidar. As massas usadas para os reagentes utilizados estão mostrados na tabela a seguir: 
Tabela 1: Quantidades, em miligramas, de Fe 3+, Fe 2+ e a massa total de Fe usadas na preparação das amostras P3E, P10 e P11.

\begin{tabular}{c|c|c|c}
\hline Amostras & $\mathrm{Fe}^{3+}(\mathrm{mg})$ & $\mathrm{Fe}^{2+}(\mathrm{mg})$ & Massa total de Fe \\
\hline P3E & 0,266 & 0,264 & 0,530 \\
\hline P10 & 0,160 & 0,160 & 0,320 \\
\hline P11 & 0,110 & 0,110 & 0,220 \\
\hline
\end{tabular}

A mistura foi agitada por 5 minutos, em um vortexer, até a homogeneização da solução. Para que fosse realizada a ligação cruzada do polímero, ao produto final foram adicionados $0,7 \mathrm{~mL}$ de glutardialdeído, e a mistura foi novamente agitada. Então, esperou-se que a solução apresentasse a aparência gelatinosa. Fato que ocorreu em poucas horas.

Após a gelatinação da amostra a mesma foi adicionada a $50 \mathrm{~mL}$ de uma solução de $0,4 \%$ de hidróxido de sódio. Ao adicionar a amostra na solução de hidróxido de sódio observa-se a mudança de cor da solução de amarelo para preto, indicando a formação da magnetita.

Após 15 min de repouso a amostra foi lavada com água destilada e centrifugada a 10.000 rpm por 5 minutos por algumas vezes, até a obtenção de pH próximo a 8. Em seguida, ocorreu a secagem da amostra a aproximadamente $100^{\circ} \mathrm{C}$.

É importante salientar que com o intuito de aumentar a concentração das partículas, quantidades diferentes da massa total de Fe foram utilizadas em cada amostra. À medida que se aumentou a massa total de Fe utilizada na preparação de determinada amostra, consequentemente aumentou-se o número de partículas da amostra em questão.

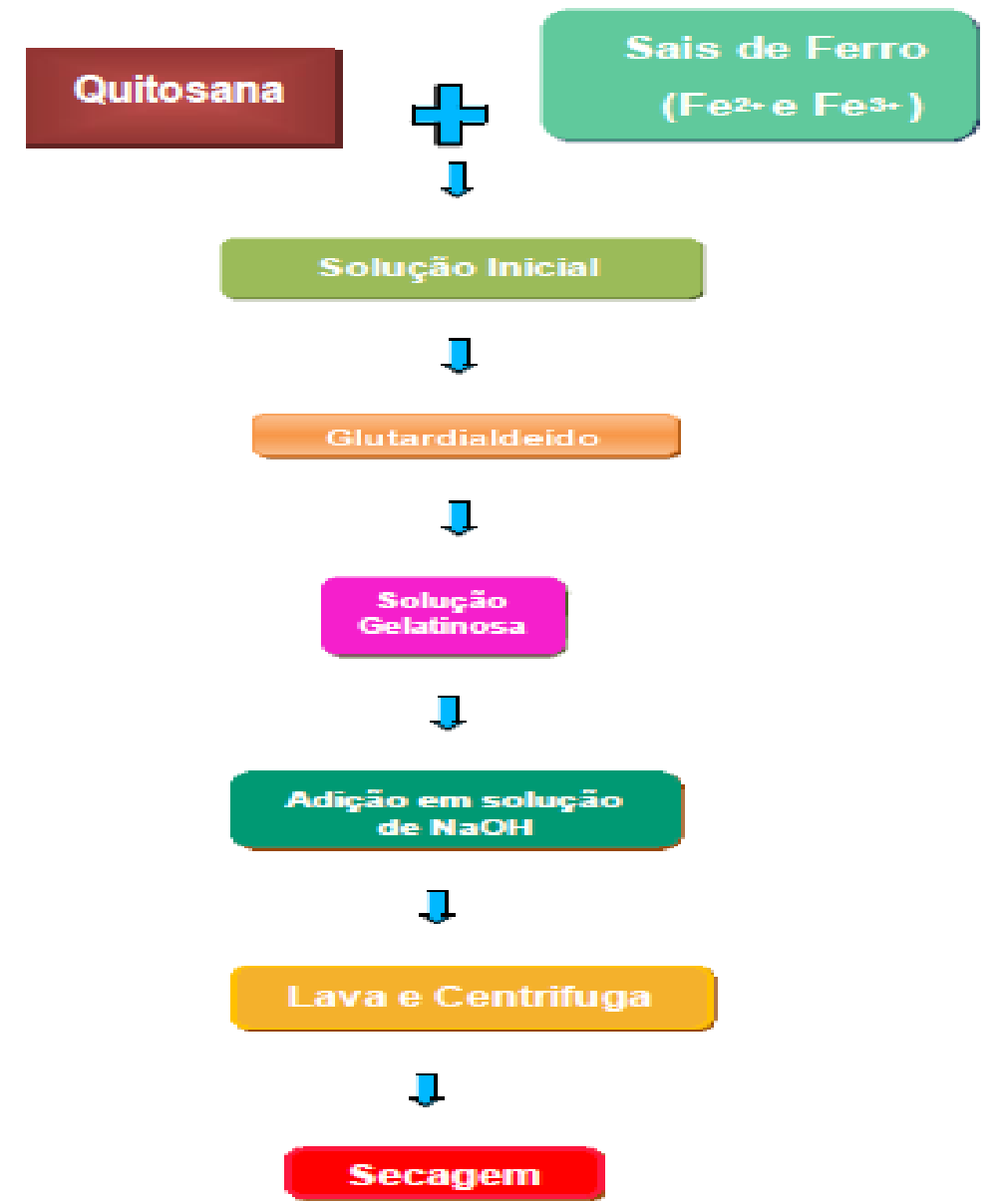

Figura 1: Esquema da síntese das nanopartículas na matriz da quitosana. 


\section{Técnicas de caracterização das amostras}

Diferentes técnicas de caracterização foram empregadas neste trabalho objetivando descrever os aspectos estruturais e magnéticos das nanopartículas de magnetita preparadas. Neste capítulo serão discutidas as técnicas de difração de raios-X, espectroscopia Mössbauer e a de Magnetização.

\subsection{Difração de raios X (DRX)}

O método dos pós foi a técnica de difração utilizada para o trabalho apresentado referente a mais frequente técnica de difração de raios-X utilizada. Um exemplo de aplicação desta técnica é a determinação do parâmetro de rede. Fator este determinado para as amostras preparadas neste trabalho.

O equipamento utilizado para a caracterização estrutural das amostras obtidas na execução deste trabalho consiste de um equipamento de Raios-X, como mostra a Figura 9, do Laboratório de ánalises ópticas e magnéticas (LAMOp) da Universidade do Estado do Rio Grande do Norte (UERN), essa infraestrutura faz parte do mestrado em Física da associação parcial entre UERN e a UFERSA.

A identificação estrutural das amostras analisadas foi feita comparando os difratogramas obtidos com padrões tabelados na literatura.

\subsection{Espectroscopia Mössbauer}

Esta técnica é bastante utilizada para investigar as formas químicas do ferro presente nas amostras, o percentual de cada fase ferruginosa, a cristalização das fases, o estado de oxidação do ferro, além do comportamento magnético (superparamagnetismo, ferro ou ferrimagnetismo, antiferromagnetismo), principal objetivo da utilização desta técnica neste trabalho.

\subsection{Medidas de Magnetização}

Para este trabalho as medidas de magnetização foram feitas utilizando as técnicas de VSM (Vibrating Sample Magnetometer) nos Laboratórios de Magnetismo do Departamento de Física da UERN. Está é uma das técnicas mais utilizadas na caracterização de materiais magnéticos. Desenvolvido por S. Foner em 1995, o VSM, é o mais utilizado nos laboratórios de pesquisa devido sua boa performance, baixo custo e simplicidade de funcionamento (Gurgel, 2010).

\section{Resultados e discussões}

Neste capítulo será apresentado os resultados obtidos a partir dos métodos de caracterização utilizados. Primeiramente, com o intuito de confirmar a formação da fase desejada e estimar o tamanho das nanopartículas e os parâmetros de rede, foi utilizado a difração de raios-X. Em seguida, foi analisado os espectros Mössbauer e as figuras de magnetização, para fins da caracterização magnética do material.

\subsection{Difração de raios-x}

Os picos que surgem nos difratogramas das amostras preparadas P10, P11 e P3E, foram comparados com as medidas da magnetita pura. Tais amostras apresentam nos difratogramas de raios- $\mathrm{X} \mathrm{a} \mathrm{Fe}_{3} \mathrm{O}_{4}$ com fase única e sem impurezas. As amostras preparadas se adequam bem àquele da magnetita pura, apresentando picos de difração em (220), (311) e (400), os índices de Miller (hkl) que indicam a formação da estrutura cristalina das partículas de magnetita. O difratograma da magnetita pura está representado na Figura 2. A Figura 3 (a), (b) e (c) exibe uma ampliação do difratograma na região $2 \theta$ entre $25^{\circ}$ e $50^{\circ}$ para as amostras realizadas para este trabalho. 


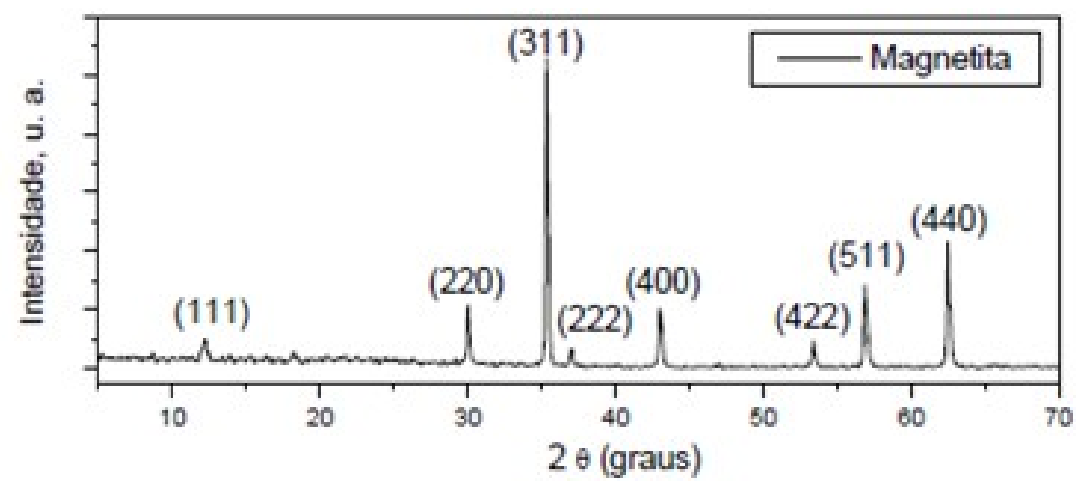

Figura 2: Difratogramas de raios-X da magnetita pura.
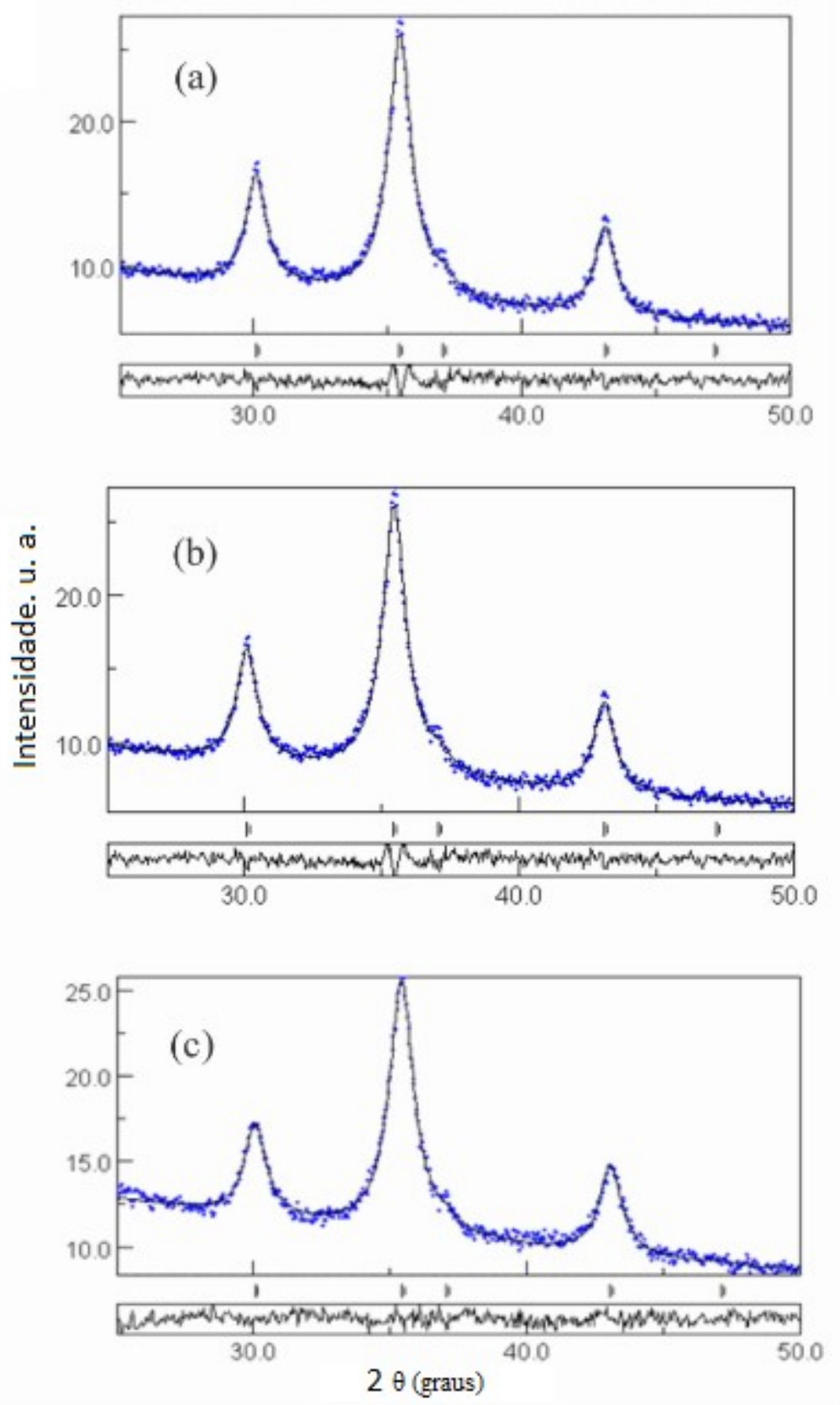

Figura 3: Difratogramas de raios X das nanopartículas (a) P3E, (b) P10 e (c) P11.

A partir dos difratogramas foi realizado o refinamento Rietveld de cada uma das amostras utilizando-se do programa MAUD (Material Analysis Using Difraction). Após o refinamento destas amostras foram obtidos o valor médio para o tamanho de partículas produzidas e os parâmetros de rede, apresentados na tabela 2, bem como tornar possível a identificação do tipo de estrutura cristalina (cúbica de faces centrada). 
As posições dos picos de difração obtidos para as amostras e o valor médio do parâmetro de rede obtido, $a=0,839 \AA$, estão em concordância com os padrões da estrutura da magnetita (Ferreira, 2009).

Tabela 2: Diâmetro, parâmetro de rede, massa total de ferro e n de partículas de cada amostra.

\begin{tabular}{c|c|c|c|c}
\hline AMOSTRAS & DIÂMETRO & $\begin{array}{c}\text { PARÂMETRO } \\
\text { DE REDE } \\
\text { (ANGSTROM) }\end{array}$ & $\begin{array}{c}\text { MASSA TOTAL } \\
\text { DE Fe (mg) }\end{array}$ & $\begin{array}{c}\mathrm{N}^{\circ} \text { DE } \\
\text { PARTÍCULAS }\end{array}$ \\
\hline P3E & 12,9 & 8,392 & 0,53 & $9,43536 * 1016$ \\
\hline P10 & 12,6 & 8,392 & 0,32 & $6,1135^{*} 1016$ \\
\hline P11 & 12,5 & 8,398 & 0,22 & $4,30471 * 1016$ \\
\hline
\end{tabular}

A reação a seguir deixa clara a proporção estequiométrica entre o $\mathrm{Fe}$ e a magnetita $\left(\mathrm{Fe}_{3} \mathrm{O}_{4}\right)$ :

$$
3 \mathrm{Fe}+4 \mathrm{H}_{2} \mathrm{O} \rightarrow \mathrm{Fe}_{3} \mathrm{O}_{4}+4 \mathrm{H}_{2}
$$

Então, primeiramente, através de análises estequiométricas foi possível definir a quantidade em mols de $\mathrm{Fe}^{2+}\left[\mathrm{Fe}\left(\mathrm{NO}_{3}\right)\right]$ e $\mathrm{Fe}^{3+}\left[\mathrm{Fe}_{2}\left(\mathrm{SO}_{4}\right)\right]$ presentes em cada amostra, a partir da quantidade em massa utilizadas destes ferros. A partir deste resultado foi encontrado a quantidade total em mols de Fe para cada amostra e, pela estequiometria da reação já citada - 3 moles de Ferro para 1 mol de magnetita - (3:1) foram utilizadas novas analises estequiométricas, de forma a encontrar a quantidade em mol de magnetita para cada amostra e posteriormente o número de partículas de cada magnetita preparada através da relação de moles de magnetita com o número de Avogadro equivalente a 6, 02x1023 átomos/mol. Logo, ao analisar a tabela 2, foi possível perceber que quanto maior a massa total de ferro utilizada na preparação da amostra, maior será o número de partículas alcançado. Além de que todas as amostras preparadas são constituídas por partículas com tamanhos bem parecidos, representando nanopartículas de magnetita.

O gráfico situado logo abaixo dos difratogramas, como mostrado na Figura 3, representa a diferença entre o ajuste feito pelo programa MAUD e os dados experimentais, quando muito acentuado a intensidade desse gráfico significa que o ajuste não ficou bem feito devido a uma escolha não adequada da base cristalográfica ou a utilização de parâmetros não adequados para iniciar o ajuste. Para este trabalho é possível perceber que a intensidade desse gráfico não se comporta de forma acentuada, logo o ajuste está bem definido. Os traços verticais situados à frente do nome magnetita na mesma Figura (Figura 3) indicam os locais onde devem aparecer os picos de acordo com o ajuste da magnetita e o local onde apareceram os picos dos dados da amostra. Os locais de surgimento dos picos do difratograma das amostras coincidem com o ajuste da magnetita.

\subsection{Espectroscopia mössbauer}

Segundo (Balasubramaniam et al., 2004), através dessa técnica de caracterização podemos investigar e analisar a distribuição de fases, o percentual de cada fase de ferro na amostra, a cristalização das fases, o comportamento magnético, incluindo o superparamagnetismo, permite confirmar as fases observadas por difração de raios X, entre outras vantagens.

Para identificar os estados superparamagnético e bloqueado é necessário ajustar os espectros com componentes dubleto (ou singleto) e sextetos e dos ajustes obtêm-se a área de absorção relativa (AAR) desses subespectros. Assim, a temperatura em que a AAR desses subspectros tem os mesmos valores é chamada de temperatura de bloqueio Mössbauer (TBM).

O superparamagnetismo é um efeito dinâmico que depende do tempo de relaxação dos momentos magnéticos, e a temperatura de bloqueio depende do tempo de medida. O tempo de medição Mössbauer é quase $10^{-10}$ ordens de grandeza menor do que o tempo de medida do VSM (medidas de magnetização). Portanto, para um sistema magnético não interagente com partículas muito pequenas, um valor maior TBM é esperada com relação ao tempo de medida do VSM, (TB). 
As partículas foram classificadas de acordo com o tipo de espectro Mössbauer observados a temperatura ambiente. Todas as amostras acusaram comportamento de partículas magneticamente bloqueadas para o tempo de medida Mössbauer (10 s), como pode ser visto na Figura 16.

Os espectros Mössbauer foram coletados utilizando o espectômetro do LAMOp, com modo de transmissão de aceleração constante e fonte de Co57 de atividade $50 \mathrm{mCi}$. O espectrômetro é equipado com um transdutor (Wissenschaftliche elektronik modelo MR 260) que varia a velocidade gerando um sinal de geometria triangular. Os deslocamentos isoméricos foram corrigidos em relação à folha de $\alpha$-Fe, padrão também utilizado para a calibração da escala de velocidade Doppler. O multicanal utilizado foi um CMCA 550, operando com 1024 canais. O detector do espectrômetro é alimentado com uma tensão de 1950 V. O programa utilizado para o ajuste dos espectros foi o Fito, desenvolvido por Jari í Hjøllum, University of Copenhagen and Risoe National Laboratory, Denmark (Galdino, 2011).
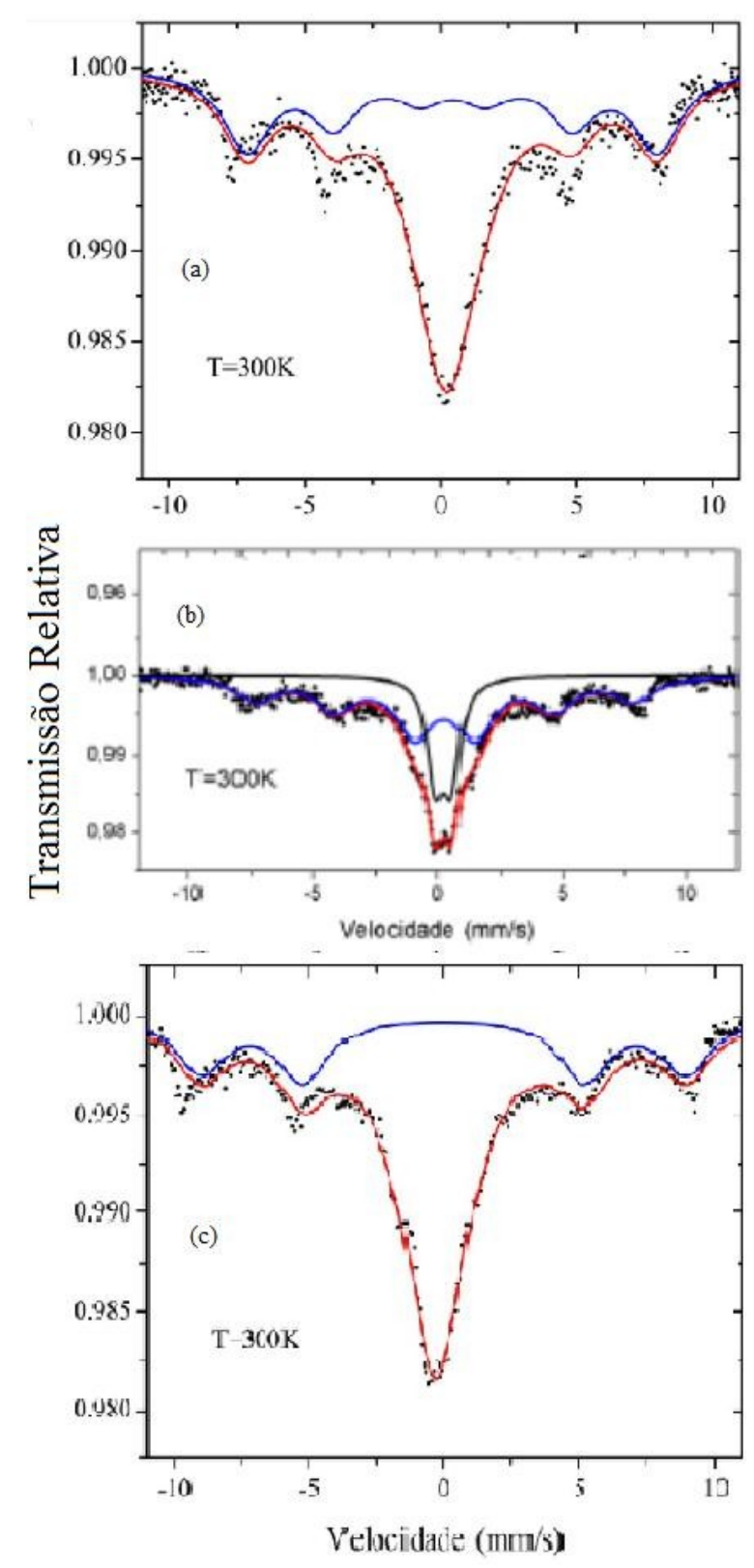

Figura 4: Espectros Mössbauer das amostras (a) P3E, (b) P10 e (c) P11. 


\subsection{Medidas de magnetização}

A Figura 5 mostra as curvas de histereses magnéticas das três amostras à temperatura ambiente.

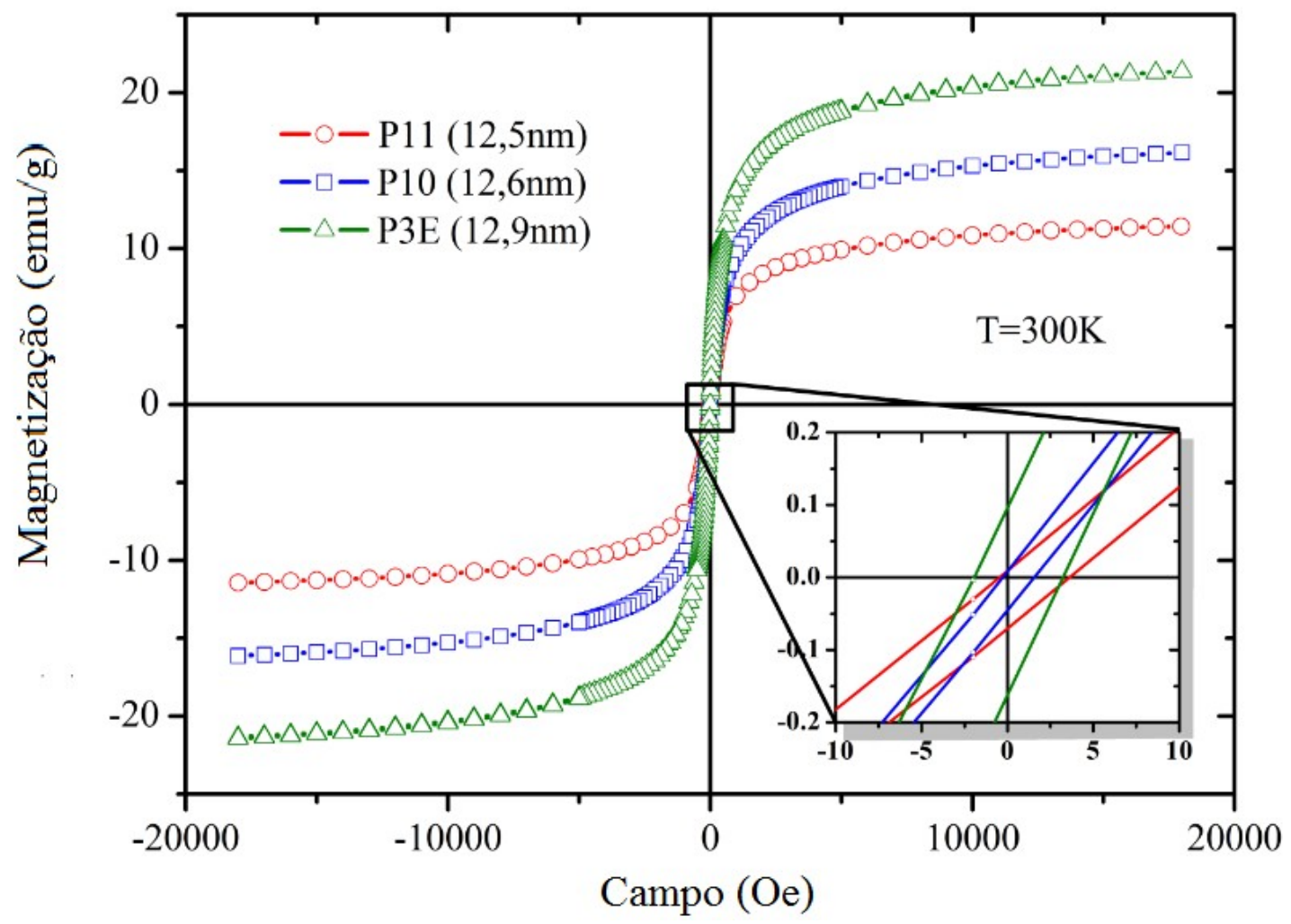

Figura 5 - Curvas de histerese das amostras P3E, P10 e P11 à temperatura ambiente.

É possível observar na Figura 5 um aumento na magnetização de saturação (MS) das amostras com o aumento da quantidade de magnetita $\left(\mathrm{Fe}_{3} \mathrm{O}_{4}\right)$. $\mathrm{O}$ valor da magnetização de saturação das amostras variaram desde 11 até $22 \mathrm{emu} / \mathrm{g}$, esses valores são menores que o valor esperado para a magnetita pura (MS = 90-95 emu/g) devido à presença do polímero quitosana. As medidas foram feitas com um passo de 20 Oe. O campo coercivo acusou valores pequenos e menores que 5 Oe indicando que todas as amostras apresentam comportamento superparamagnético, para o tempo de medida da magnetização (10-8 s).

magnetização remanente (MR) obtida quando, ao diminuir a intensidade do campo $\mathrm{H}$, a curva toca o eixo das ordenadas, ou seja, assim que o campo aplicado é desligado. Neste ponto a magnetização não é nula, logo um grande número de domínios estão ainda alinhados. O valor de MS refere-se à magnetização medida quando o campo aplicado é máximo. E a razão MR /MS nos diz o quanto magnético é um material, quanto mais próximo de 1 (um) essa razão estiver mais momentos magnéticos permaneceram orientados após a retirada do campo externo e menor será a perda na orientação dos momentos. O campo coercivo (HC) trata-se da propriedade onde a magnetização é zero.

Das curvas de magnetização mostradas na Figura 5 foi possível obter os valores dos parâmetros MS, MR, HC e MR /MS. Estes valores podem ser vistos na Tabela 3: 
Tabela 3: Valores dos parâmetros MS, MR, HC e MR /MS obtidos das nanopartículas preparadas P11, P10 e P3E.

\begin{tabular}{c|c|c|c|c}
\hline Amostra & MS (emu/g) & MR (emu/g) & HC (Oe) & MR /MS \\
\hline P11 & 11,5 & 0.39 & 1.8 & 0,03 \\
\hline P10 & 17 & 0.02 & 0.9 & 0,001 \\
\hline P3E & 22 & 0.12 & 2.6 & 0,005 \\
\hline
\end{tabular}

\section{Conclusões}

Através dos resultados das medidas feitas nas amostras de nanopartículas de magnetita realizadas, foram obtidas as seguintes afirmações:

Foi possível aumentar a densidade das nanopartículas, mantendo constante o tamanho, controlando a quantidade de reagentes, aumentando-se a quantidade de massa total de Fe nas amostras.

As medidas de difração de raios-X mostraram que as nanopartículas possuem valores médios do parâmetro de rede de 0,839 Angstrom, característicos da magnetita pura, e diâmetro médio de $12,7 \mathrm{~nm}$.

As medidas de espectroscopia Mössbauer feitas em temperatura ambiente mostraram que todas as amostras estão magneticamente bloqueadas, para o tempo de medida Mössbauer.

Através das medidas de magnetização foi possível observar um aumento na magnetização de saturação (MS) de acordo com o aumento da quantidade de magnetita $\left(\mathrm{Fe}_{3} \mathrm{O}_{4}\right)$ preparada. Como as amostras possuem tamanhos muito próximos, as curvas de histerese das amostras apresentaram valores dos parâmetros MR e MR /MS próximos de zero, já que os valores de HC indicaram que as amostras têm características de um material superparamagnético, para o tempo de medida da magntezição.

\section{Perspectivas}

- A utilização de outras formas de caracterização magnética das amostras, com o objetivo de buscar resultados cada vez mais precisos e positivos sobre a utilidade das nanopartículas de magnetita em técnicas de tratamento, como a hipertermia.

- Estudar propriedades de aquecimento das partículas em presença do campo magnético alternado.

- Pesquisar as vantagens desenvolvidas por essas nanopartículas quando as partículas estiverem muito próximas e interagindo magneticamente.

Synthesis of magnetite nanoparticles with diameters controlled aiming at applications in hyperthermia

Albstract: In this presentation we show a method for the preparation of magnetite nanoparticles with diameter and density controlled particle. Nanoparticles were synthesized in the chitosan matrix, two sets of samples were prepared. The density of the nanoparticles was increased by controlling the amount of reactants. Measurements of X-ray diffraction showed that the nanoparticles have a lattice parameter of $0.839 \AA$ and have an average diameter of $12.6 \mathrm{~nm}$. Images of transmission electron microscopy showed monodisperse particles with very narrow log-normal size distribution. The obtained network parameter shows that nanoparticles are of high purity. Mössbauer spectroscopy measurements, 
performed at room temperature showed spectra due to magnetically blocked particles, and this effect mainly due to the magnetic interaction between the nanoparticles.

Keywords: Magnetic hyperthermia; magnetism; magnetite.

\section{Referências bibliográficas}

ARANTES, F. R. Estudo do comportamento magnético de nanopartículas de magnetita e nanofios de níquel diluídos em cristais líquidos liotrópicos. São Paulo, 2010.

SOUZA, K. C. Síntese e Caracterização de Nanopartículas e Nanocompósitos Magnéticos para Aplicações Biomédicas.Belo Horizonte, 2011.

ZHANG, G.; LIAO, Y.; BAKER, I. Surface engineering of core/ shell iron/ iron oxide nanoparticles from microemulsions for hyperthermia. Materials Science and nanoparticles from microemulsions for hyperthermia. Materials Science and Engineering: C, Elsevier, v. 30, n. 1, p. 92-97, 2010. 2, 42

GALDINO, V. B., Estudo dos acoplamentos magnéticos em nanopatículas CoFe2O4/CoFe2 com estrutura de núcleo-camada, Dissertação de Mestrado, Universidade do Estado do Rio Grande do Norte, 2011. 